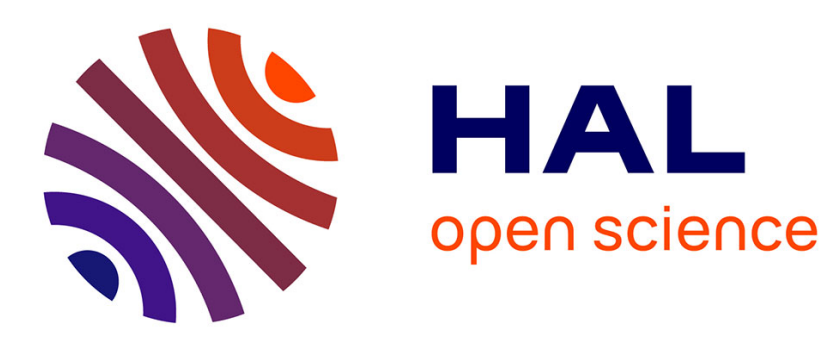

\title{
Approximating Event System Abstractions by Covering their States and Transitions
}

Jacques Julliand, Olga Kouchnarenko, Pierre-Alain Masson, Guillaume Voiron

\section{To cite this version:}

Jacques Julliand, Olga Kouchnarenko, Pierre-Alain Masson, Guillaume Voiron. Approximating Event System Abstractions by Covering their States and Transitions: Version 1. [Research Report] RRFEMTO-ST-2496, FEMTO-ST. 2017. hal-01496470

\section{HAL Id: hal-01496470 \\ https://hal.science/hal-01496470}

Submitted on 27 Mar 2017

HAL is a multi-disciplinary open access archive for the deposit and dissemination of scientific research documents, whether they are published or not. The documents may come from teaching and research institutions in France or abroad, or from public or private research centers.
L'archive ouverte pluridisciplinaire HAL, est destinée au dépôt et à la diffusion de documents scientifiques de niveau recherche, publiés ou non, émanant des établissements d'enseignement et de recherche français ou étrangers, des laboratoires publics ou privés. 


\section{INSTITUT FEMTO-ST}

\section{Approximating Event System Abstractions by Covering their States and Transitions}

\section{Version 1}

Jacques Julliand — Olga Kouchnarenko — Pierre-Alain Masson — Guillaume Voiron

Rapport de Recherche n $^{\circ}$ RR-FEMTO-ST-2496

DÉPARTEMENT DISC - March 9, 2017 



\title{
femto-st \\ imters \\ TECHNOLOGIES
}

\section{Approximating Event System Abstractions by Covering their States and Transitions}

Version 1

\author{
Jacques Julliand, Olga Kouchnarenko, Pierre-Alain Masson, Guillaume Voiron \\ Département DISC \\ Vesontio \\ Rapport de Recherche no RR -FEMTO-ST-2496 March 9, 2017 ( pages)
}

\begin{abstract}
In event systems, contrarily to sequential ones, the control flow is implicit. Consequently, their abstraction may give rise to disconnected and unreachable paths. This paper presents an algorithmic method for computing a reachable and connected under-approximation of the abstraction of a system specified as an event system. We compute the under-approximation with concrete instances of the abstract transitions, that cover all the states and transitions of the predicate-based abstraction. To be of interest, these concrete transitions have to be reachable and connected to each other. We propose an algorithmic method that instantiates each of the abstract transitions, with heuristics to favour their connectivity. The idea is to prolong whenever possible the already reached sequences of concrete transitions, and to parameterize the order in which the states and actions occur. The paper also reports on an implementation, which permits to provide experimental results confirming the interest of the approach with related heuristics.
\end{abstract}

Key-words: Predicate abstraction; under-approximation; event systems

FEMTO-ST Institute, DISC research department

UFR Sciences - Route de Gray - F-25030 BESANÇON cedex FRANCE

Tel: (33 3) 81666515 - Fax: (33 3) 81666450 - e-mail: fvernill@femto-st.fr 



\title{
Approximation d'abstractions de systèmes d'événements en couvrant leurs états et transitions
}

\author{
Version 1
}

Résumé : Dans les systèmes d'événements, contrairement aux systèmes séquentiels, le flot de contrôle est implicite. En conséquence, leur abstraction peut conduire à des chemins non connexes et innateignables. Ce papier présente une méthode algorithmique permettant le calcul d'une sousapproximation, connexe et atteignable, de l'abstraction d'un système d'événements. Notre sousapproximation est constituée d'instances concrètes des transitions abstraites, et couvre tous les états et transitions d'une abstraction par prédicats. Pour être exploitables, ces instances concrètes doivent être atteignables et connexes entre elles. Nous proposons un algorithme qui instancie chaque transition abstraite, avec des heuristiques visant à favoriser leur connexité. Le principe est de prolonger chaque fois que c'est possible les séquences d'instances concrètes déjà atteintes, et de paramétrer l'ordre dans lequel les états et les événements sont considérés. Ce papier décrit aussi une implantation de la méthode, qui nous a permis d'obtenir des résultats expérimentaux confirmant l'intérêt de la méthode.

Mots-clés : Abstraction par prédicats; sous-approximation; systèmes d'événements 



\title{
Approximating Event System Abstractions by Covering their States and Transitions
}

\author{
Jacques Julliand, Olga Kouchnarenko , Pierre-Alain Masson, Guillaume Voiron \\ FEMTO-ST, UMR 6174 CNRS and Univ. Bourgogne Franche-Comté \\ 16, route de Gray F-25030 Besançon Cedex France \\ \{jjulliand, okouchna, pamasson, gvoiron\}@femto-st.fr
}

March 9, 2017

\begin{abstract}
In event systems, contrarily to sequential ones, the control flow is implicit. Consequently, their abstraction may give rise to disconnected and unreachable paths. This paper presents an algorithmic method for computing a reachable and connected under-approximation of the abstraction of a system specified as an event system. We compute the under-approximation with concrete instances of the abstract transitions, that cover all the states and transitions of the predicate-based abstraction. To be of interest, these concrete transitions have to be reachable and connected to each other. We propose an algorithmic method that instantiates each of the abstract transitions, with heuristics to favour their connectivity. The idea is to prolong whenever possible the already reached sequences of concrete transitions, and to parameterize the order in which the states and actions occur. The paper also reports on an implementation, which permits to provide experimental results confirming the interest of the approach with related heuristics.
\end{abstract}

Keywords. Predicate abstraction; under-approximation; event systems

\section{Introduction}

Abstracting a program or its specification allows to control the size of its state space description, at the price of a loss in accuracy. That facilitates their algorithmic exploitation, otherwise limited by the huge if not infinite number of concrete states. The general idea of abstraction is to gather states that share common properties into super-states. In predicate abstraction [GS97] the concrete states are mapped onto a finite set of abstract ones, by means of a set of predicates that characterizes each abstract state. An abstract transition links two abstract states when it has at least one concrete instantiation. Such transitions are called may [GJ03, meaning that they may be instantiated. Still there is no guarantee that two consecutive may transitions can necessarily be instantiated as two consecutive connected concrete transitions: their respective target and source concrete states may differ.

This paper aims at computing connected and reachable concrete paths from a predicate abstraction of a system formally specified as an event system Abr10, which is a special kind of action system [Dij75, Dij76]. We propose an algorithmic method for computing an underapproximation that covers all the states and transitions of this abstraction.

An event in an event system specifies state variable modifications by means of a guarded action. The actions are activated whenever their guard becomes true, so that there is no natural control flow as in a program. As a result, paths of the system may become disconnected and even unreachable in the abstraction. Still, we are interested in covering the reachable part 
of the abstraction as best as possible. This work has been motivated by a testing purpose: our intention is to cover paths of an abstraction by tests. But the method could also apply for example to the model-checking of safety properties. These potential applications are not presented in this paper due to lack of space.

We propose to under-approximate the abstraction by computing concrete instances of the abstract event sequences. The idea behind our method is to favour the connectivity and reachability of the successive concrete instances by prolonging whenever possible the already reached concrete transitions. Our proposal in this paper is as sketched:

- we instantiate each of the abstract transitions by enumerating all the possibilities of connecting two abstract states by any event,

- we use heuristics for controlling the order in which the events and states are enumerated, according to some know-how of the natural flow of the events succession,

- we use concrete state coloration, similarly to [VY03, for prolonging preferably the sequences known to be reachable and connected.

Our contributions allow then generating a concrete transition system from an event system. We also report on an implementation, which permits us to provide experimental results confirming the interest of the approach with the related heuristics.

The technical background of our paper regarding event systems, predicate abstraction and may transition systems is given in Sec. 2. Section 3 presents an electrical system as an illustrative example. The algorithm for computing both an abstraction and its approximation is presented in Sec. 4. The heuristics that we propose to enhance the coverage achieved by the algorithm are given in Sec. 5. Our experimental results are presented in Sec. 6. Section 7 describes related work, and Sec. 8 concludes the paper.

\section{Background}

In this paper systems are specified by event systems (ES) described in the B syntax Abr96, Abr10 1. Notice however that our proposals and results are generic since event system semantics is defined by concrete labelled transition systems.

In this section we first present the syntax and the semantics of the B event systems. Then we present the concept of predicate abstraction and formalize the abstraction of event systems by means of May Transition Systems (MTS).

\subsection{Model Syntax and Semantics}

We start by introducing B event systems in Def. 1. The events are defined by means of guarded actions Dij75 by composition of five primitive actions where $E, F$ are arithmetic expressions and $P, P^{\prime}$ are predicates: skip an action with no effect, $x, y:=E, F$ a multiple assignment, $P \Rightarrow a$ a guarded action, $a_{1}[] a_{2}$ a bounded non-deterministic choice between $a_{1}$ and $a_{2}$, and @ z.a an unbounded non-deterministic choice $a_{z_{1}}[] a_{z_{2}}[] \ldots$ for all the values of $z$ satisfying the guard of $a$ denoted as $\operatorname{grd}(a)$. Here grd is defined on the primitive actions by: $\operatorname{grd}($ skip) $\stackrel{\text { def }}{=}$ true, $\operatorname{grd}(x, y:=E, F) \stackrel{\text { def }}{=}$ true, $\operatorname{grd}\left(P^{\prime} \Rightarrow a\right) \stackrel{\text { def }}{=} P^{\prime} \wedge \operatorname{grd}(a), \operatorname{grd}\left(a_{1}[] a_{2}\right) \stackrel{\text { def }}{=} \operatorname{grd}\left(a_{1}\right) \vee \operatorname{grd}\left(a_{2}\right)$ and $\operatorname{grd}(@ z . a) \stackrel{\text { def }}{=} \exists z \cdot \operatorname{grd}(a)$.

\footnotetext{
${ }^{1}$ We could alternatively use a syntax with guarded commands Dij75, such as Abstract State Machines GKOT00, Gur00.
} 
Definition 1 (Event System) Let Ev be a set of event names. A B event system is a tuple $\langle X, I$, Init, EvDef $\rangle$ where $X$ is a set of state variables, $I$ is a state invariant, Init is an initialization action such that $I$ holds in any initial state, EvDef is a set of event definitions, each in the shape of $e \stackrel{\text { def }}{=} a$ for any $e \in \mathrm{Ev}$, and such that every event preserves $I$.

Figure 1(b) depicts an example of an event system that illustrates Def. 1. Following [BC00], we use labelled transition systems to define the semantics of event systems.

Let $e \stackrel{\text { def }}{=} a$ be an event. It has a weakest precondition Dij76 and a weakest conjugate precondition [BC00 w.r.t. a set of target states $Q^{\prime}$ denoted respectively as $w p\left(a, Q^{\prime}\right)$ and $w c p\left(a, Q^{\prime}\right) . w p\left(a, Q^{\prime}\right)$ is the largest set of states from which applying $a$ always leads to a state of $Q^{\prime}$ whereas $w c p\left(a, Q^{\prime}\right)$ is the largest set of states from which it is possible to reach a state of $Q^{\prime}$ by applying $a$. An event also defines a relation between the values of the state variables $\mathrm{X}$ before and after the application of the event. It is expressed by the before after predicate of the event $e \stackrel{\text { def }}{=} a$ denoted as $\operatorname{prd}_{X}(a)$.

Let us now formally define $w p, w c p$ and $p r d_{X}$ following [BJM16. Basically, we directly consider the set of states $Q$ and $Q^{\prime}$ as predicates of the same name. We define the $w p$ w.r.t. the five primitive actions as:

- $w p\left(\operatorname{skip}, Q^{\prime}\right) \stackrel{\text { def }}{=} Q^{\prime}$,

- $w p\left(x:=E, Q^{\prime}\right) \stackrel{\text { def }}{=} Q^{\prime}[E / x]$ that is the usual substitution of $x$ by $E$ as in Hoa69],

- $w p\left(P \Rightarrow a, Q^{\prime}\right) \stackrel{\text { def }}{=} P \Rightarrow w p\left(a, Q^{\prime}\right)$,

- $w p\left(a_{1}[] a_{2}, Q^{\prime}\right) \stackrel{\text { def }}{=} w p\left(a_{1}, Q^{\prime}\right) \wedge w p\left(a_{2}, Q^{\prime}\right)$,

- $w p\left(@ z . a, Q^{\prime}\right) \stackrel{\text { def }}{=} \forall z . w p\left(a, Q^{\prime}\right)$ where $z$ is not free in $Q^{\prime}$.

We define the $w c p$ and $p r d_{X}$ w.r.t. $w p$ as:

- $w c p\left(a, Q^{\prime}\right) \stackrel{\text { def }}{=} \neg w p\left(a, \neg Q^{\prime}\right)$,

- $\operatorname{prd}_{X}(a) \stackrel{\text { def }}{=} w c p\left(a, x_{1}^{\prime}=x_{1} \wedge \ldots \wedge x_{n}^{\prime}=x_{n}\right)$ that is a predicate on the state variables $X=$ $\left\{x_{1}, \ldots, x_{n}\right\}$ in the source state before $a$ and the target state variables $X^{\prime}=\left\{x_{1}^{\prime}, \ldots, x_{n}^{\prime}\right\}$ after $a$.

\subsection{Predicate Abstraction}

Predicate abstraction [GS97 is a special instance of the framework of abstract interpretation CC92 that maps the potentially infinite state space $C$ of a CTS onto the finite state space $\mathbf{A}$ of an abstract transition system via a set of $n$ predicates $\mathcal{P} \stackrel{\text { def }}{=}\left\{p_{1}, p_{2}, \ldots, p_{n}\right\}$ over the state variables. The set of abstract states A contains $2^{n}$ states. Each state is a tuple $q \stackrel{\text { def }}{=}\left(q_{1}, q_{2}, \ldots, q_{n}\right)$ with $q_{i}$ being equal either to $p_{i}$ or to $\neg p_{i}$, and we also consider $q$ as the predicate $\bigwedge_{i=1}^{n} q_{i}$. We define a total abstraction function $\alpha: C \rightarrow \mathrm{A}$ such that $\alpha(c)$ is an abstract state $q$ where $c$ satisfies $q_{i}$ for all $i \in 1 . . n$. By a misuse of language, we say that $c$ is in $q$, or that $c$ is a concrete state of $q$.

Let us now define the abstract transitions as may ones. Consider two abstract states $q$ and $q^{\prime}$ and an event $e$. There exists a may transition from $q$ to $q^{\prime}$ by $e$, denoted by $q \stackrel{e}{\rightarrow} q^{\prime}$, if and only if there exists at least one concrete transition $c \stackrel{e}{\rightarrow} c^{\prime}$ where $c$ and $c^{\prime}$ are concrete states with $\alpha(c)=q$ and $\alpha\left(c^{\prime}\right)=q^{\prime}$. 
Let us define for any predicate $P$ the solver call $S A T(P)$, which returns either a model satisfying $P$ or unsat if $P$ is unsatisfiable or unknown if the solver couldn't determine the satisfiability of P. Let $e \stackrel{\text { def }}{=} a$ be an event definition, $q \stackrel{e}{\rightarrow} q^{\prime}$ is a may transition iff $S A T\left(w c p\left(a, q^{\prime}\right) \wedge q\right)$. We compute a concrete witness $c \stackrel{e}{\rightarrow} c^{\prime}$ by using the before after predicate: $\left(c, c^{\prime}\right):=S A T\left(\operatorname{prd}_{X}(a) \wedge\right.$ $\left.q^{\prime}\left[X^{\prime} / X\right] \wedge q\right)$ where $q^{\prime}\left[X^{\prime} / X\right]$ is the predicate $q^{\prime}$ in which each state variable $x_{i}$ is substituted by $x_{i}^{\prime}$.

\subsection{May Transition Systems}

Let us introduce may transition systems (MTS), which are transition systems with abstract states, and abstract may transitions. They are closely related to Modal Transition Systems defined in [LT88, GHJ01, Bal04].

Definition 2 (May Transition System) Let Ev be a finite set of event names and $\mathcal{P} \stackrel{\text { def }}{=}$ $\left\{p_{1}, p_{2}, \ldots, p_{n}\right\}$ be a set of predicates. Let $A$ be a finite set of abstract states defined by $\left\{p_{1}, \neg p_{1}\right\} \times$ $\left\{p_{2}, \neg p_{2}\right\} \times \ldots \times\left\{p_{n}, \neg p_{n}\right\}$. A tuple $\left\langle Q, Q_{0}, \Delta\right\rangle$ is an MTS if it satisfies the following conditions:

- $Q(\subseteq A)$ is a finite set of states,

- $Q_{0}(\subseteq Q)$ is a set of abstract initial states,

- $\Delta(\subseteq Q \times \mathrm{Ev} \times Q)$ is a may labelled transition relation.

Now, Definition 3 associates an abstraction defined by an MTS with an event system.

Definition 3 (MTS associated with an ES) Let ES $\stackrel{\text { def }}{=}\langle X, I$, Init, EvDef $\rangle$ be an event system and $\mathcal{P} \stackrel{\text { def }}{=}\left\{p_{1}, p_{2}, \ldots, p_{n}\right\}$ be a set of $n$ predicates over variables of $X$ defining a set of $2^{n}$ abstract states $A \stackrel{\text { def }}{=}\left\{p_{1}, \neg p_{1}\right\} \times\left\{p_{2}, \neg p_{2}\right\} \times \ldots \times\left\{p_{n}, \neg p_{n}\right\}$. A tuple $\left\langle Q, Q_{0}, \Delta\right\rangle$ is an MTS associated to $E S$ and $\mathcal{P}$ if it satisfies the following conditions:

- $Q \stackrel{\text { def }}{=}\left\{q \in A \mid \exists\left(q^{\prime}, e\right) .\left(q \stackrel{e}{\rightarrow} q^{\prime} \in \Delta \vee q^{\prime} \stackrel{e}{\rightarrow} q \in \Delta\right)\right\}$,

- $Q_{0} \stackrel{\text { def }}{=}\{q \mid q \in A \wedge S A T(\operatorname{sp}($ Init,$I) \wedge q)\}$,

- $\Delta \stackrel{\text { def }}{=}\left\{q \stackrel{e}{\rightarrow} q^{\prime} \mid q \in A \wedge q^{\prime} \in A \wedge e \stackrel{\text { def }}{=} a \in \operatorname{EvDef} \wedge S A T\left(w c p\left(a, q^{\prime}\right) \wedge q\right)\right\}$.

An example of an MTS, whose ES is described in Fig. 1(b), can be seen in Fig. 2 (in dashed lines). The four abstract states named $q_{0}$ to $q_{3}$ appear as rounded rectangular dashed boxes. The predicates $p_{1}$ and $p_{2}$ from which they are defined are explicitly given in Sec. 3. The abstract transitions of $\Delta$ are represented as dashed arrows labelled by event names.

\section{Illustrative Example: an Electrical System}

To illustrate our approach, this section describes an electrical (EL) system example. It is a finite state control and command system that illustrates the MTS, as represented in Fig. 2 .

Figure 1(a) shows a device $D$ powered via a switch to one of three batteries $B_{1}, B_{2}$, and $B_{3}$. A clock $H$ periodically sends a signal that causes a commutation of the closed switch. The system has to meet the following requirements: one and only one switch is closed at a time, and a clock signal changes the switch that is closed. The batteries may break down. If it happens to the one that is powering $D$, an exceptional commutation is triggered. We assume that there is 


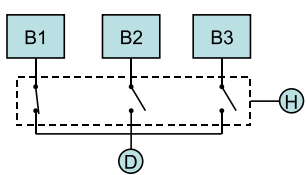

(a) Physical Representation

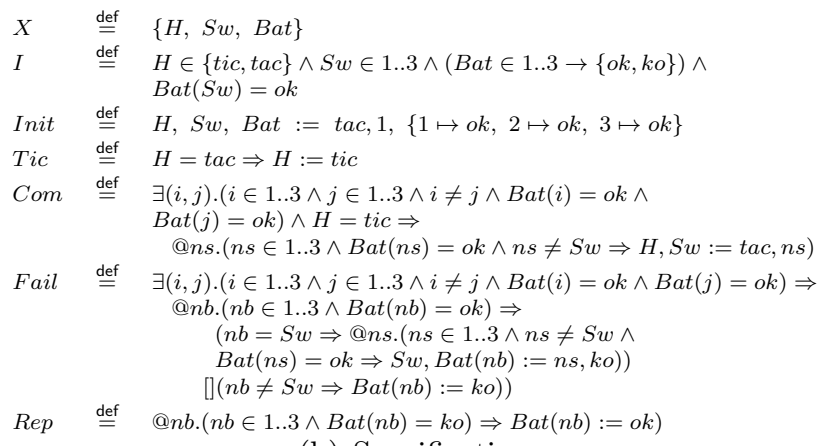

(b) Specification

Figure 1: Electrical system and its specification

always at least one battery working. When there is only one battery working, the clock signals are ignored.

The event system in Fig. 1(b) uses three variables. $H$ models the clock and takes two values: tic to ask for a commutation, and tac when it has occurred. $S w$ models the switches by indicating which one is closed. Bat models the batteries breakdowns by a total function that associates $o k$ or $k o$ (for a broken battery) to each battery. The state changes occur by applying four events: Tic sends a commutation signal, Com changes the closed switch responding to a Tic, Fail breaks down at random a battery, and Rep repairs at random a broken battery.

The part in dashed lines in Fig. 2 shows the MTS that abstracts the model of Fig. 1(b) w.r.t. the set $\mathcal{P}_{0} \stackrel{\text { def }}{=}\left\{p_{1}, p_{2}\right\}$ of abstraction predicates, where $p_{1} \stackrel{\text { def }}{=} H=t i c$ and $p_{2} \stackrel{\text { def }}{=} \exists(i, j) .(i \in$ $1 . .3 \wedge j \in 1 . .3 \wedge i \neq j \wedge \operatorname{Bat}(i)=o k \wedge \operatorname{Bat}(j)=o k)$.

\section{Abstraction and Approximated Transition System Computa- tion}

This section presents an algorithm used to compute both an abstraction that is an MTS, and an under-approximation. The reunion of both is called an Approximated Transition System (see Def. (4), in which $\left\langle C, C_{0}, \Delta^{c}\right\rangle$ is an under-approximation of the labelled transition system that is the semantics of the event system from which the MTS is deduced. In the rest of the paper, for the abstract states, we distinguish between the may-reachability and the reachability. The former is the reachability by the abstract may transition relation $\Delta$, and the latter is the reachability by the concrete transition relation $\Delta^{c}$ in the ATS. We say that an abstract state $q$ is reachable if there exists at least one concrete instance of $q$ that is reachable thanks to the transition relation $\Delta^{c}$. By extension, an abstract transition is reachable if there exists at least one concrete instance in $\Delta^{c}$ whose source state is reachable. The concretization of the may transitions is performed on the fly during the computation of the MTS. This algorithm guarantees that every may transition between two abstract states is concretized. A total abstraction function $\alpha$ maps each concrete state of $C$ to an abstract state of $Q$.

The algorithm comes in two versions, both of them being presented in the same figure due to a lack of room. The first version is the one presented in this section. It is referred to as Alg. 1. The second version, referred to as Alg. 2, is enhanced by heuristics that are explained in Sec. 5. The differences between Alg. 1 and Alg. 2 are highlighted and enclosed in square brackets. Read the left hand highlighted parts for Alg. 1, and replace them with the right hand ones for Alg. 2. Notice that since Alg. 2 computes more things than Alg. 1, some fictive empty parts (the empty highlighted square brackets [ ]) have been added in Alg. 1. 
Definition 4 (Approximated Transition System) Let $\left\langle Q, Q_{0}, \Delta\right\rangle$ be an MTS. A tuple $\langle Q$, $\left.Q_{0}, \Delta, C, C_{0}, \alpha, \Delta^{c}\right\rangle$ is an ATS whose $\left\langle C, C_{0}, \alpha, \Delta^{c}\right\rangle$ is a concretization of the MTS $\left\langle Q, Q_{0}\right.$, $\Delta\rangle$ where:

- $C, C_{0}$ are sets of respectively concrete states and concrete initial states,

- $\alpha$ is a total abstraction function from $C$ to $Q$,

- $\Delta^{c}(\subseteq C \times E v \times C)$ is a concrete labelled transition relation.

Figure 2 shows the ATS computed by Alg. 2 for the electrical system of Fig. 1(a) with predicates $\mathcal{P}_{0}$ (see Sec. 3). The MTS appears in dashed lines while the full lines represent its concretization. The concrete states are showed as big dots numbered according to their order of discovery by Alg. 2 .

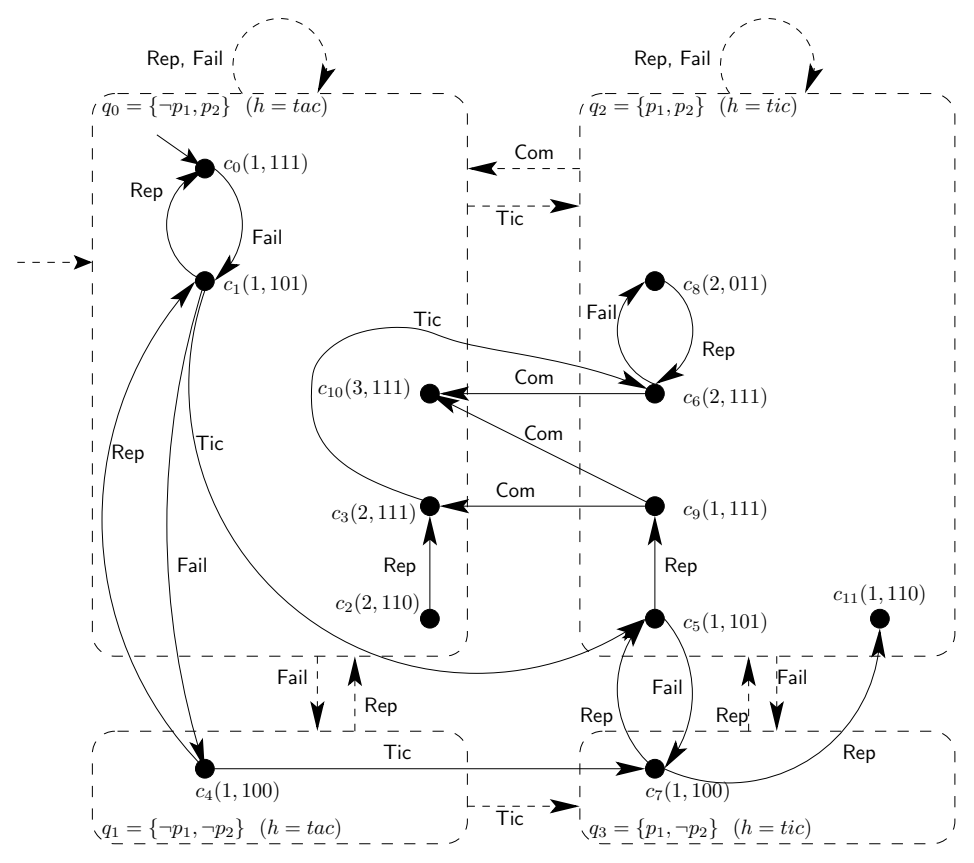

Figure 2: Example MTS and ATS of the Electrical System. The values of the concrete states are indicated in parentheses right by them. For example with state $c_{8},(2,011)$ means that battery 2 is used, and that battery 1 is $k o$ while batteries 2 and 3 are $o k$. The value of $h$ is given globally in the abstract states.

Lines 1 to 8 of Alg. 1 compute the set of initial abstract states $Q_{0}$, an instance of each being recorded as a concrete witness in $C_{0}$ with its association in $\alpha$. Lines 9 to 34 compute the may transition relation $\Delta$. Each abstract transition is concretized by a witness $\left\{c_{w} \stackrel{\mathrm{e}}{\rightarrow} c_{w}^{\prime}\right\}$, and the concrete states $c_{w}$ and $c_{w}^{\prime}$ are recorded in $C$ with their associations in $\alpha$. For that it computes in the set $R Q$ the set of may-reachable states. For each may-reachable source state, it checks for each potential abstract state (line 12) and for each event (line 13) if a may transition exists (line 14). When it is the case, the algorithm records the witness transition (see lines 16 and 27 ), but also possibly another concrete transition (see lines 17 to 26) whose source state is one of the existing concrete states of the current source state $q$ when it exists. This last transition is computed first to improve the reachability of the concrete transition relation. Indeed, the existing concrete states are more likely to be connected to the initial states than the witness source state provided by the solver in line 14. Last, line 30 adds $q^{\prime}$ as a may-reachable state that has not been taken into account yet to compute the may transition relation. 


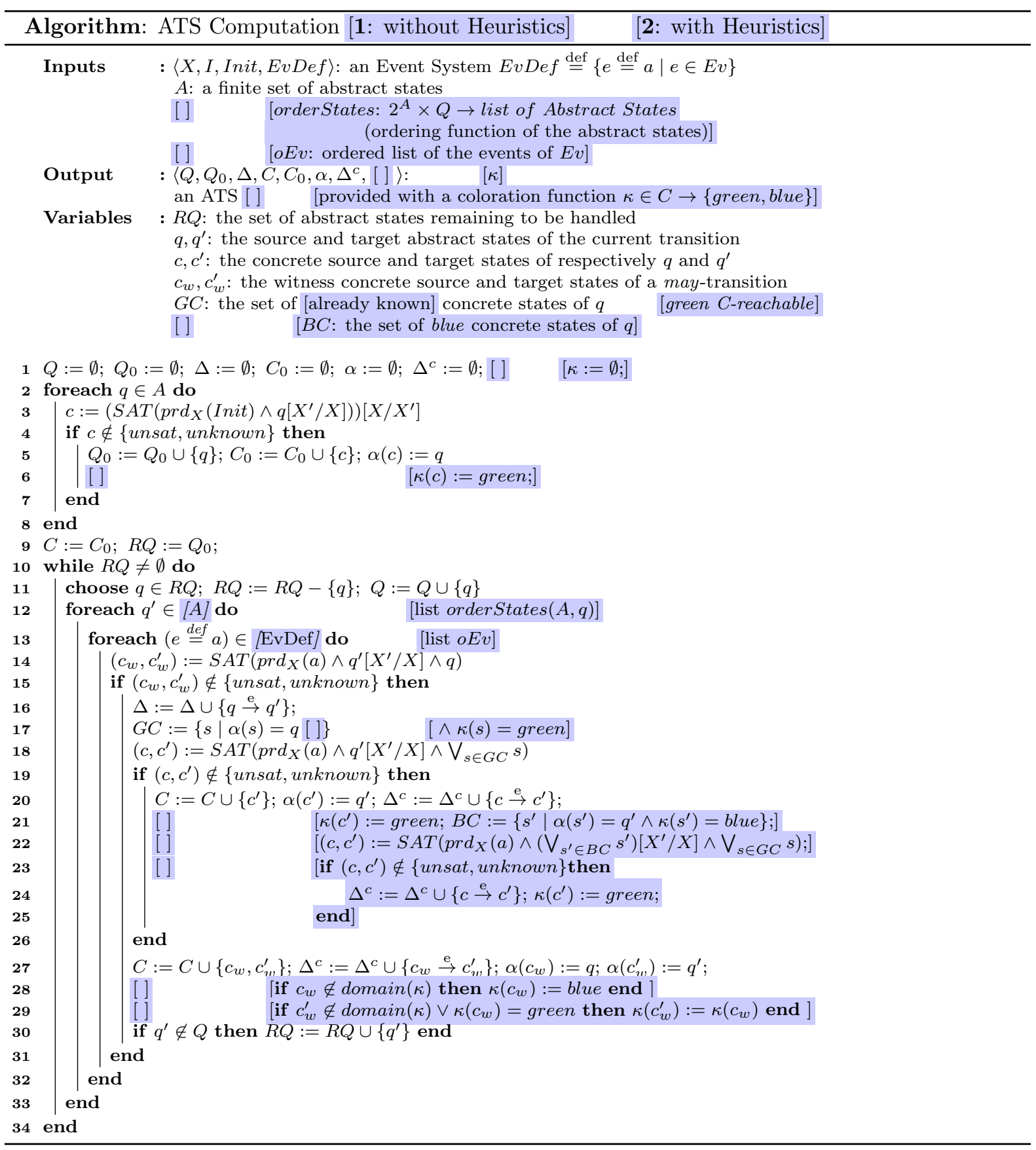

\section{Heuristics for Better Abstraction Coverage}

Using Alg. 1, the connectivity and the reachability of the computed ATS might be weak, since it strongly depends on the solver's choices. This section provides two heuristics, integrated into Alg. 2, for improving both the connectivity and the reachability. The first heuristic addresses this problem by allowing the engineer to firstly define an order for the set of events of $E v$ in an ordered list $o E v$ (line 13 ) and, secondly, a custom function ordering the set of abstract states A (line 12). The second heuristic, exposed in Sec. 5.2, adapts the partial computation of reachability proposed in VY03 to our purpose for integrating it into Alg. 2. The resulting new algorithm's complexity is the same as the previous one. 


\subsection{Events and States Ordering}

Our first heuristic consists of providing means to control the order in which the events and abstract target states are handled by the algorithm.

Indeed, usually in reactive systems, some events can only be fired after other events have previously been executed. Let us consider the EL system where no battery repairing (modelled by the Rep event) can occur unless at least one battery has broken down first (modelled by the Fail event). Since Alg. 1 currently uses an unordered set of events EvDef, it might attempt to concretize a Rep transition before trying to concretize any Fail transition. In this case, the concrete source state of the Rep transition would not be a reachable one. To fix this, we introduce the ordered list of events $o E v$ as an input in Alg. 2. To compute a complete abstraction, i.e. covering all events and all states, $o E v$ must contain at least one occurrence of each event of the set EvDef. For example, for the EL system, in all judicious orders, Fail must precede Rep for the aforementioned reason, and Tic must precede Com because Com is a response to the event Tic.

Similarly, the orderStates function parameterizes Alg. 2. Thanks to this function, the order in which the abstract target states are handled can be controlled. To compute a complete abstraction, the list returned by orderStates must contain at least all the abstract states of A. While being completely customizable by the engineer, the function used in our experiments presented in Sec. 6 gives better results for an order in which the first target abstract state handled is the source abstract state (state $q$ in line 11) and the other states are ordered arbitrarily. Indeed, treating reflexive abstract transitions first tends to increase the number of reachable concrete states within the source abstract state. As a result, the chances that the next abstract transitions can be concretized from a reachable source state are increased.

When applying Alg. 1 to the EL system with a set of abstraction predicates (first AP in Table 1), $33.33 \%$ of the abstract states and $11.11 \%$ (see line 1) of the abstract transitions are covered by the ATS. Integrating the event and state ordering without coloration improved these ratios respectively to $66.67 \%$ and $44.44 \%$.

These ordering heuristics are integrated into Alg. 2, along with the concrete states coloration discussed in Sec. 5.2. Even though our results did not focus on that point, an interesting perspective could be to consider the concretization of a same abstract transition multiple times. This could be useful for instance for systems requiring initialization steps that repetitively apply the same event, such as a credit card system for example. In fact, this behaviour can already be implemented using our algorithm by adding the same event to $o E v$ several times, and by adding the target state of the abstract transition several times to the list returned by the orderStates function.

\subsection{Concrete States Coloration}

The reachability of the concrete states of the under-approximation is improved and computed on the fly in Alg. 2 without increasing its complexity w.r.t. Alg. 1. The principle is to associate a colour with each concrete state. A reachable state is coloured in green and a state whose reachability is unknown is coloured in blue. While Alg. 1 tried to concretize the abstract transitions from an already known concrete state, Alg. 2 uses the reachability information when concretizing the abstract transitions. It first tries to concretize an abstract transition from any known and reachable state (see lines 17 and 18). If it is indeed possible (line 19), the solver returns a first reachable concrete transition, added to the ATS, whose concrete target state becomes green (see lines 20 and 21). To improve the connectivity, the algorithm also tries to join a green source concrete state to a target blue one, whose reachability is thus currently unknown (line 22). If it is possible (line 23), its colour becomes green (line 24) since it is a 
target of a concrete transition starting from a reachable (green) concrete state. Even if the concretization from a known green concrete state is not possible, the abstract transition is still concretized. The corresponding concrete source and target states may already be known. In this case, their reachability remain the same. Otherwise, since we have no information about their reachability, they are coloured in blue (see lines 28 and 29).

When applying Alg. 2 with coloration and without events and states ordering to the EL system with the first set of abstraction predicates in Table 1. 100\% of the abstract states and $77.78 \%$ of the abstract transitions are covered by the ATS. This is better than with Alg. 1 that gives respectively $33.33 \%$ and $11.11 \%$. Moreover, integrating the two heuristics seen in Sec. 5.1 into Alg. 1 improved these two ratios to $100 \%$.

These heuristics allow improving the reachability of the ATS for all the systems that we use in our experiments (see Sec. 6.2).

\section{Implementation and Experimentation}

This section introduces in Sec. 6.1 our proof-of-concept tool developed to evaluate the effects of the heuristics. The experimental results on four examples in Table 1 are presented in Sec. 6.2. Then, in Sec. 6.3 we analyse these results and conclude on the contributions of the heuristics presented in this paper.

\subsection{About the Tool}

The developed tool can be seen as a library for handling abstract and concrete transition systems as well as event systems. It embeds an event-B parser and allows to manipulate most event-B systems. The two algorithms are implemented and can be applied to them. The library also provides the user with many facilities for dealing with event systems. For instance, pre-implemented functions allow to easily compute an abstraction of a model from a set of abstraction predicates, as well as the $w p$, wcp and before-after predicates $p r d_{X}$ of events defined by guarded actions. The library also contains functions to check the modality of abstract transitions and to find a concretization of an abstract state or an abstract transition. It can also be seen as a simple API for multiple SMT-solvers since the tool automatically generates SMT-Lib2 code for checking the satisfiability of any first order boolean formula. The tool is constituted of more than 5000 lines of JAVA code (version 8) and uses Z3 [dMB08] as default SMT-solver. The library can be downloaded at https://github. com/stratosphr/stratest/ wiki. This website also gives information on how to use the tool.

\subsection{Experimental Results}

This section provides the results obtained when applying Alg. 1 and Alg. 2 to a set of four realistic event systems of increasing size. These event systems were taken back from various previous work without modification so as not to influence the experiment and threaten the validity of the results.

The set of examples contains the electrical system (EL) presented in Sec. 3, a phone book service $(\mathbf{P H})$, a coffee machine system $(\mathbf{C M})$, and a car alarm system $(\mathbf{C A})$. For each of them, two different sets of abstraction predicates have been used (see the AP column).

The following column names appear in Table 1: Sys for the system studied and an upper approximation of its size between parentheses, \#Ev for the number of events in the event system, AP for an identification of the set of abstraction predicates used, \# AP for the number 


\begin{tabular}{|c|c|c|c|c|c|c|c|c|c|c|c|c|c|}
\hline Sys & \#Ev & AP & \#AP & Alg. & \#As & 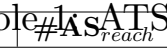 & $\mathrm{C}_{A} \mathrm{~S}(\mathrm{a}) \mathrm{A}$ & )ा & RAqults & $\tau_{A T}(\%)$ & \#CT & $\rho_{C T}$ & Time \\
\hline \multirow{4}{*}{$\begin{array}{c}\text { EL } \\
(24)\end{array}$} & \multirow{4}{*}{4} & \multirow{2}{*}{1} & \multirow[b]{2}{*}{2} & 1 & 3 & 1 & 33.33 & \begin{tabular}{l|}
9 \\
\end{tabular} & 1 & 11.11 & 13 & 13 & $00: 00.283$ \\
\hline & & & & 2 & 3 & 3 & 100 & 9 & 9 & 100 & 18 & 2 & $00: 00.297$ \\
\hline & & \multirow{2}{*}{2} & \multirow{2}{*}{2} & 1 & 4 & 4 & 100 & 11 & 8 & 72.73 & 15 & 1.88 & 00:00.429 \\
\hline & & & & 2 & 4 & 4 & 100 & 11 & 11 & 100 & 17 & 1.55 & 00:00.449 \\
\hline \multirow{4}{*}{$\begin{array}{c}\mathbf{P H} \\
\left(2^{10}\right)\end{array}$} & \multirow{4}{*}{4} & \multirow{2}{*}{1} & \multirow{2}{*}{3} & 1 & 3 & 3 & 100 & 12 & 11 & 91.67 & 16 & 1.45 & $00: 00.261$ \\
\hline & & & & 2 & 3 & 3 & 100 & 12 & 12 & 100 & 22 & 1.83 & $00: 00.287$ \\
\hline & & \multirow{2}{*}{2} & \multirow{2}{*}{6} & 1 & 8 & 8 & 100 & 62 & 60 & 96.77 & 83 & 1.38 & 00:01.994 \\
\hline & & & & 2 & 8 & 8 & 100 & 62 & 62 & 100 & 88 & 1.42 & 00:02.204 \\
\hline \multirow{4}{*}{$\begin{array}{l}\text { CM } \\
\left(2^{16}\right)\end{array}$} & \multirow{4}{*}{8} & \multirow{2}{*}{1} & \multirow[b]{2}{*}{3} & 1 & 4 & 3 & 75 & 30 & 5 & 16.67 & 47 & 9.4 & $00: 00.753$ \\
\hline & & & & 2 & 4 & 4 & 100 & 30 & 24 & 80 & 54 & 2.25 & $00: 00.854$ \\
\hline & & \multirow[b]{2}{*}{2} & \multirow[b]{2}{*}{3} & 1 & 6 & 3 & 50 & 52 & 7 & 13.46 & 83 & 11.86 & 00:01.639 \\
\hline & & & & 2 & 6 & 6 & 100 & 52 & 25 & 48.08 & 77 & 3.08 & 00:01.629 \\
\hline \multirow{4}{*}{$\begin{array}{c}\text { CA } \\
\left(2^{15}\right)\end{array}$} & \multirow{4}{*}{20} & \multirow{2}{*}{1} & \multirow[b]{2}{*}{0} & 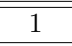 & 8 & 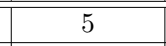 & 62.5 & 31 & 18 & 58.065 & 44 & 2.44 & $00: 13.818$ \\
\hline & & & & 2 & 8 & 8 & 100 & 31 & 25 & 80.65 & 50 & 2 & $00: 13.283$ \\
\hline & & \multirow[b]{2}{*}{2} & \multirow[b]{2}{*}{9} & 1 & 9 & 5 & 55.56 & 30 & 11 & 36.67 & 37 & 3.36 & $00: 23.978$ \\
\hline & & & & 2 & 9 & 9 & 100 & 30 & 28 & 93.33 & 52 & 1.86 & $00: 25.381$ \\
\hline
\end{tabular}

of abstraction predicates in $\mathbf{A P}, \mathbf{A l g}$. for the algorithm applied, \#AS for the number of mayreachable abstract states, \#A $\mathbf{A} \mathbf{S}_{\text {reach }}$ for the number of reachable abstract states computed, $\tau_{A S}$ for the abstract state coverage that is the ratio $\frac{\# A S_{\text {reach }}}{\# A S}$, \#AT for the number of abstract transitions, \# $\mathbf{A} \mathbf{T}_{\text {reach }}$ for the number of reachable abstract transitions computed. Note that we say that an abstract transition is reachable if there exists a concrete instance of it in the ATS that is reachable. Next, there are the following column names: $\tau_{A T}$ for the abstract transition coverage that is the ratio $\frac{\# A T_{\text {reach }}}{\# A T}$, \#CT for the number of concrete transitions computed, $\rho_{C T}$ for the ratio $\frac{\# C T}{\# A T_{\text {reach }}}$ which measures the efficiency of the method, by indicating in average how many concrete transitions have been computed for making an abstract transition reachable, and finally Time for the ATS computation runtime (in minutes). The connectivity between transitions is indirectly measured via the coverage and efficiency rates, since a reachable state or transition is necessarily connected to a concrete initial state.

The main results of our method are the coverage ratios of abstract states $\left(\tau_{A S}\right)$ and abstract transitions $\left(\tau_{A T}\right)$. For almost identical computation time(s), an improvement of these ratios indicates a better performance of the method. For $\rho_{C T}$, a value between 3 and 1 indicates that the algorithm covers one abstract transition per iteration step. When this ratio decreases that indicates an improvement of the efficiency. Indeed, for each abstract transition, each iteration step computes one up to three transitions according to the conditions in lines 19 and 23. For the EL system, with the first set of abstraction predicates, $\rho_{C T}$ decreases from 13 to 2 , meaning that the heuristic allowed to compute more interesting concrete transitions, increasing the abstraction transition coverage from $11.11 \%$ to $100 \%$.

\subsection{Analysis of the Obtained Results}

This section gives some conclusions and insights about the results exposed in Table 1.

As expected, the ATS computation times are nearly identical, no matter which version of the algorithm has been used. Note that for two cases out of eight the ATS computation time with Alg. 2 is on average slightly faster than with Alg. 1. Since the formulas whose satisfiability is checked are different between the two algorithms, the solver may be faster to provide an answer for the formulas in Alg. 2 than in Alg. 1.

We observe that Alg. 2 improves both the abstraction coverage rates and the efficiency $\rho_{C T}$ compared to Alg. 1. In particular, we point out the $\mathbf{C M}$ case with the first $\mathbf{A P}$ where the transition coverage and the efficiency achieved by Alg. 2 is about respectively five and four times better than by Alg. 1 . 
For all systems and all sets of abstraction predicates, the abstraction coverage is improved by Alg. 2. Depending on the set of predicates used, the coverages for states and transitions can reach up to 100\%. On most examples however, Alg. 1 covers less than half of the abstract states and transitions. Note for example the $\mathbf{C M}$ case with the second set of abstraction predicates where the abstract states and transitions coverage(s) are respectively twice and three times better using the heuristics. All these results empirically confirm the interest of the proposed heuristics to improve the abstraction coverage.

The heuristics also produced good results concerning the efficiency rate $\rho_{C T}$. For most cases, its value is decreased by Alg. 2 thanks to the heuristics, which means that they generally help concretizing abstract transitions by useful transitions improving the abstraction coverage. For the $\mathbf{C M}$ system with the second AP and Alg. 1 for example, an average of 11.86 concrete transitions need to be computed in order to cover one abstract transition. When the heuristics are used however, an average of only 3.08 concrete transitions computation is needed to cover one abstract transition.

The ordering heuristic alone does not necessarily improve the abstraction coverage w.r.t. Alg. 1, whereas the coloration heuristic alone always improves the results. Nevertheless, for four cases out of the eight presented in this paper, combining the ordering and coloration heuristics improves the abstraction coverage compared to coloration only. For the CM system with the first AP for example, the ordering heuristic alone covers two abstract states compared to three with Alg. 1, and six abstract transitions compared to five. The coloration heuristics alone covered all of the four abstract states, and twenty-two out of the thirty abstract transitions. When combining both heuristics, the four abstract states and twenty-four abstract transitions are covered.

\section{Related Work}

In [NK00] and in [PPV07, the set of abstraction predicates is iteratively refined in order to compute a bisimulation of the semantics of the model when it exists. None of these two methods is guaranteed to terminate, because of the refinement step that sometimes needs to be repeated

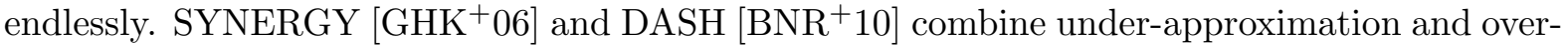
approximation computations to check safety properties on programs. As we aim at proposing an efficient method to build a reachable under-approximation of a system that covers all abstract states and all abstract transitions w.r.t. a specification and a set of predicates, our algorithm does not refine the approximation and so always terminates.

The closest methods to ours are those that are proposed in GGSV02] and in [VY03]. These approaches propose algorithms that compute an under approximated concretization of a predicate abstraction covering its abstract states and transitions. Both these methods are exploited for generating tests. The algorithm in GGSV02 does not traverse nor compute the may abstraction. It builds a partial concretization of the abstract states that are reached from an initial concrete state by a forward walk. To improve this method, the algorithm in [VY03] computes exhaustively the may abstraction by random abstract state generation. Therefore, some generated concrete states are not reached. Then Veanes and al. VY03 propose to distinguish between four kinds of abstract transitions: green transitions when there exists an instance that is reached from an initial concrete state, blue transitions when there exists instances, but none known to be reachable from an initial state, red transitions when there does not exist any instance, and grey transitions for the transitions that have not been concretized yet. In our method, we compute and concretize only the part of the may abstraction that is may-reachable by an abstract transition from an initial abstract state. We do not record the red transitions that are non-existing transitions in the MTS, and we do not need the grey transitions that are 
the ones which remain to be treated. In contrast with [VY03, our method colours the concrete states instead of the abstract transitions. This allows us to distinguish between the reached states (green) and the states for which we do not know whether they are reached (blue) or not. So for improving the method, Alg. 2 connects in priority a green source state $s$ to a blue target state $t$. That has a "domino effect" because all the blue reached states from $t$ remain blue, but become reachable.

Other works are about generating an under approximation to generate tests from abstraction. The tools Agatha [RGLG03, DART [GKS05], CUTE [SMA05], EXE [CGP ${ }^{+}$06] and PEX [TdH08] also compute abstractions from models or programs, but only by means of symbolic executions [PV09. This data abstraction approach computes an execution graph. Its set of abstract states is possibly infinite whereas it is finite with our method.

Our method can be applied to generate tests as the concolic execution in [SMA05. The concolic method allows to generate structural tests of systems covering partially the control flow that must be explicited. Our approach allows to generate tests covering the paths defined by the set of abstraction predicates for systems whose control flow is implicitly defined.

\section{Conclusion and Further Work}

This paper has presented an algorithmic method for computing a concrete approximation of the predicate abstraction of an event system. All of the abstract states and transitions are covered, but as the control flow is implicit in an event system, our method focuses on computing concrete sequences that are connected and reachable. We have presented two heuristics allowing us to better reach and connect these sequences. One heuristic colours the states that are known to be reachable, and the other takes a user defined order on the events and abstract states enumeration as parameters. Experimental results on four case studies are exhibited to confirm the practical interest of our approach.

As future work, we intend to define other means for guiding the sequences instantiation, in addition to the events ordering. We could introduce a relevance function on concrete states, as is done in GGSV02, for targeting peculiar concrete states considered as more relevant. Also, our intention is to use the concrete sequences that we compute as model-based tests issued from a formal model of the specification. Abstracting this model would allow selection criteria such as paths selection to be used, when the size of the explicit model would prevent it.

\section{References}

[Abr96] J.-R. Abrial. The B Book. Cambridge Univ. Press, 1996.

[Abr10] J.-R. Abrial. Modeling in Event-B: System and Software Design. Cambridge Univ. Press, 2010.

[Bal04] T. Ball. A theory of predicate-complete test coverage and generation. In FMCO, volume 3657 of $L N C S$, pages 1-22, 2004.

[BC00] Didier Bert and Francis Cave. Construction of finite labelled transition systems from B abstract systems. In IFM, pages 235-254, 2000.

[BJM16] Hadrien Bride, Jacques Julliand, and Pierre-Alain Masson. Tri-modal underapproximation for test generation. Science of Computer Programming, 132(P2):190208, 2016. 
$\left[\mathrm{BNR}^{+} 10\right]$ Nels E. Beckman, Aditya V. Nori, Sriram K. Rajamani, Robert J. Simmons, SaiDeep Tetali, and Aditya V. Thakur. Proofs from tests. IEEE Trans. Software Eng., 36(4):495-508, 2010.

[CC92] Patrick Cousot and Radhia Cousot. Abstract interpretation frameworks. J. Log. Comput., 2(4):511-547, 1992.

[CGP ${ }^{+}$06] Cristian Cadar, Vijay Ganesh, Peter M. Pawlowski, David L. Dill, and Dawson R. Engler. EXE: automatically generating inputs of death. In $A C M C C S$, pages $322-$ $335,2006$.

[Dij75] E.W. Dijkstra. Guarded commands, nondeterminacy, and formal derivation of programs. Com. of the ACM, 18(8):453-457, 1975.

[Dij76] E.W. Dijkstra. A Discipline of Programming. Prentice-Hall, 1976.

[dMB08] L. de Moura and N. Bjorner. An efficient SMT solver. In TACAS, volume 4963 of LNCS, pages 337-340, 2008.

[GGSV02] Wolfgang Grieskamp, Yuri Gurevich, Wolfram Schulte, and Margus Veanes. Generating finite state machines from abstract state machines. In ISSTA, pages 112-122, 2002 .

[GHJ01] Patrice Godefroid, Michael Huth, and Radha Jagadeesan. Abstraction-based model checking using modal transition systems. In CONCUR, pages 426-440, 2001.

$\left[\mathrm{GHK}^{+} 06\right]$ Bhargav S. Gulavani, Thomas A. Henzinger, Yamini Kannan, Aditya V. Nori, and Sriram K. Rajamani. Synergy: a new algorithm for property checking. In SIGSOFT FSE, pages 117-127, 2006.

[GJ03] P. Godefroid and R. Jagadeesan. On the expressiveness of 3-valued models. In VMCAI, volume 2575 of LNCS, pages 206-222. Springer, 2003.

[GKOT00] Yuri Gurevich, Philipp W. Kutter, Martin Odersky, and Lothar Thiele. Abstract State Machines, Theory and Applications, volume 1912 of LNCS. Springer, 2000.

[GKS05] Patrice Godefroid, Nils Klarlund, and Koushik Sen. DART: directed automated random testing. In PLDI, pages 213-223, 2005.

[GS97] S. Graf and H. Saidi. Construction of abstract state graphs with PVS. In $C A V$, volume 1254 of $L N C S$, pages 72-83. Springer, 1997.

[Gur00] Yuri Gurevich. Sequential abstract-state machines capture sequential algorithms. ACM Trans. Comput. Log., 1(1):77-111, 2000.

[Hoa69] C.A.R. Hoare. An axiomatic basis for computer programming. Com. of the ACM, 12(10):576-580, 1969.

[LT88] Kim Guldstrand Larsen and Bent Thomsen. A modal process logic. In LICS, pages 203-210, 1988.

[NK00] K. S. Namjoshi and R. P. Kurshan. Syntactic program transformations for automatic abstraction. In $C A V$, volume 1855 of $L N C S$, pages 435-449, 2000.

[PPV07] Corina S. Păsăreanu, Radek Pelánek, and Willem Visser. Predicate abstraction with under-approximation refinement. LMCS, 3(1:5):1-22, 2007. 
[PV09] Corina S. Păsăreanu and Willem Visser. A survey of new trends in symbolic execution for software testing and analysis. STTT, 11(4):339-353, 2009.

[RGLG03] N. Rapin, C. Gaston, A. Lapitre, and J.-P. Gallois. Behavioral unfolding of formal specifications based on communicating extended automata. In ATVA, 2003.

[SMA05] Koushik Sen, Darko Marinov, and Gul Agha. CUTE: a concolic unit testing engine for C. In ESEC/SIGSOFT FSE, pages 263-272, 2005.

[TdH08] Nikolai Tillmann and Jonathan de Halleux. Pex-white box test generation for .net. In TAP, volume 4966 of $L N C S$, pages 134-153, 2008.

[VY03] Margus Veanes and Rostislav Yavorsky. Combined algorithm for approximating a finite state abstraction of a large system. In ICSE 2003/Scenarios Workshop, pages 86-91, 2003. 



\section{fento-st - $\square$ SIENCES \& TECHNOLOGIES}

FEMTO-ST INSTITUTE, headquarters

15B Avenue des Montboucons - F-25030 Besançon Cedex France

Tel: (33 3) 63082400 - e-mail: contact@femto-st.fr

FEMTO-ST - AS2M: TEMIS, 24 rue Alain Savary, F-25000 Besançon France

FEMTO-ST - DISC: UFR Sciences - Route de Gray - F-25030 Besançon cedex France

FEMTO-ST - ENERGIE: Parc Technologique, 2 Av. Jean Moulin, Rue des entrepreneurs, F-90000 Belfort France

FEMTO-ST - MEC'APPLI: 24, chemin de l'épitaphe - F-25000 Besançon France

FEMTO-ST - MN2S: 15B Avenue des Montboucons - F-25030 Besançon cedex France

FEMTO-ST - OPTIQUE: 15B Avenue des Montboucons - F-25030 Besançon cedex France

FEMTO-ST - TEMPS-FREQUENCE: 26, Chemin de l'Epitaphe - F-25030 Besançon cedex France

http://www.femto-st.fr 